\title{
FLAT MANIFOLDS WITH PARALLEL TORSION
}

\author{
F. W. KAMBER \& PH. TONDEUR
}

1. Consider a linear connection on a smooth manifold. The connection is flat, if the curvature tensor $R$ is zero. If the torsion tensor $T$ has vanishing covariant derivative, the torsion is said to be parallel. A linear connection is complete, if every geodesic can be defined for any real value of the affine parameter. In this note the following structure theorem for smooth manifolds admitting a complete flat connection with parallel torsion is proved: Any such manifold is the orbit space of a simply connected Lie group $G$ under a properly discontinuous and fixed-point free action of a subgroup of the affine group of $G$. This Theorem includes the classical cases of flat Riemannian manifolds and flat affine manifolds (Auslander and Markus [2]), where the torsion is assumed to be zero and $G$ turns out to be $\boldsymbol{R}^{n}$, and also generalizes a theorem of Hicks [9, Theorem 6] for complete connections with trivial holonomy group and parallel torsion tensor, stating that a manifold with such a connection is homogeneous. We consider the case where the curvature vanishes, without requiring the holonomy group to be trivial. In the last section we study the homotopy group of flat manifolds with parallel torsion and give characterizations for such manifolds to be Eilenberg-MacLane spaces of type $K(\pi, 1)$.

2. Let $G$ be a Lie group and Aut $G$ the group of continuous automorphisms of $G$. The affine group of $G$ [1] is the semi-direct product $A(G)=$ $G \cdot$ Aut $G$ with the multiplication $\left(g_{1}, \alpha_{1}\right) \cdot\left(g_{2}, \alpha_{2}\right)=\left(g_{1} \alpha_{1}\left(g_{2}\right), \alpha_{1} \alpha_{2}\right)$ for $g_{1}, g_{2} \in G$ and $\alpha_{1}, \alpha_{2} \in$ Aut $G$, It has a Lie group structure, and acts on $G$ by $(g, \alpha) \cdot x$ $=g \alpha(x)$ for $(g, \alpha) \in A(G), x \in G$. In the case of the additive vector group $\boldsymbol{R}^{n}$ this is the affine group $A(n)=\boldsymbol{R}^{n} \cdot G L(n, \boldsymbol{R})$ with its standard action on $\boldsymbol{R}^{n}$.

Let $G$ be connected and consider the linear connection on $G$ defined by the left invariant vector fields [8], [9]. The parallel transport is the effect of the left translations on the tangent vectors of $G$, and hence clearly independent of paths; the connection is thus flat. The geodesics through the identity element $e \in G$ are the 1-parameter subgroups of $G$ and thus defined for any real value of the affine parameter. All geodesics are translates of geodesics through $e$ and thus the connection is complete. The parallelity of the torsion is easily checked [7], [9].

Communicated by S. S. Chern, February 19, 1968. The first author was supported by a NSF grant at the Institute for Advanced Study, and the second author partially by NSF grant GP-7528. 
Consider now the Lie group $\operatorname{Aff}(G)$ of connection-preserving diffeomorphisms of $G$. The action of $A(G)$ on $G$ defines a homomorphism $\iota: A(G) \rightarrow \operatorname{Aff}(G)$.

Proposition 2.1. ' is an isomorphism.

Proof. Injectivity is clear. To prove surjectivity, let $\tau \in \operatorname{Aff}(G)$. Suppose that $\tau(e)=g$ and $\alpha(x)=g^{-1}(x)$ for all $x \in G$. Then $\alpha: G \rightarrow G$ is a connection preserving diffeomorphism with $\alpha(e)=e$. It suffices to show $\alpha \in$ Aut $G$, because then $(g, \alpha) \cdot x=g \alpha(x)=\tau(x)$ and thus $\iota(g, \alpha)=\tau . \alpha$ is connectionpreserving and therefore $d \alpha(T(X, Y))=T(d \alpha X, d \alpha Y)$ for any vector fields $X, Y$ on $G$. Now the bracket operation in the Lie algebra $g$ can be expressed through the torsion by $[X, Y]=-T(X, Y)$ for $X, Y \in \mathfrak{g}$, [7], [8] (this contains the parallelity of the torsion). It follows that $d \alpha: \mathrm{g} \rightarrow \mathrm{g}$ is a Lie algebra homomorphism and hence $\alpha \in$ Aut $G$.

q.e.d.

In the case $G=R^{n}$, this Proposition is just the fact that $A(n)$ is the full group of connection-preserving diffeomorphisms of $\boldsymbol{R}^{n}$.

3. Let $G$ be connected and simply connected and $\pi \subset A(G)$ a subgroup operating properly discontinuously and without fixed points on $G$, i.e. for each $x \in G$ there exists a neighborhood $U(x)$ such that $g_{1} U(x) \cap g_{2} U(x) \neq \varnothing$ for $g_{1}, g_{2} \in \pi$ implies $g_{1}=g_{2}$. The orbit space $\pi \backslash G$ is a smooth manifold with fundamental group $\pi$ and inherits a complete flat connection with parallel torsion (local conditions are preserved). Whereas the holonomy group of the connection on $G$ is trivial, the holonomy representation of the induced connection on $\pi \backslash G$ is the composition

$$
\pi \subseteq A(\mathrm{G}) \rightarrow \text { Aut } G \rightarrow \text { Aut } g .
$$

Here $A(G) \rightarrow$ Aut $G$ is the projection onto the second factor, and Aut $G \rightarrow$ Aut $g$ is given by the differential. Moreover $g$ is identified with the tangent space of $p(e) \in \pi \backslash G$, where $p: G \rightarrow \pi \backslash G$ is the canonical projection. With these notations (3.1) is more precisely the holonomy representation at $p(e)$. The tangent bundle of $\pi \backslash G$ is then given by

$$
T(\pi \backslash G) \cong G \times{ }_{\pi} \mathrm{g},
$$

where $\pi$ acts on $g$ by (3.1) and on $G \times g$ by $\tau(g, X)=(\tau \cdot g, \tau X)$. Aut $G \rightarrow$ Aut $g$ is injective, so that the representation (3.1) is trivial if and only if $\pi \subseteq A(G) \rightarrow$ Aut $G$ is trivial, i.e. if and only if $\pi$ consists only of translations. In this case $\pi$ is a subgroup of $G$ and the orbit space is the homogeneous space $\pi \backslash G$ of right cosets of $G \bmod \pi$. By a result of Hicks [9, Theorem 6], any manifold with complete flat connection and parallel torsion is such a homogeneous space, provided the holonomy group is zero. We generalize this as follows.

Structure Theorem 3.3. Let $M$ be a smooth connected manifold. Then $M$ admits a complete flat connection with parallel torsion if and only if there are a connected and simply connected Lie group $G$, a subgroup $\pi \subset A(G)$ acting 
properly discontinuously and without fixed points on $G$, and a connectionpreserving diffeomorphism $M \cong \pi \backslash G$.

Proof. We have shown that the condition is sufficient. The necessity is seen as follows. The universal covering $\bar{M}$ has a complete flat connection with parallel torsion and trivial holonomy group. The invariant vector fields on $\vec{M}$ with respect to this parallel transport form a Lie algebra $g$ with respect to the usual bracket. Let $G$ be the unique connected and simply connected Lie group with Lie algebra $g$, equipped with the connection defined by the left invariant vector fields. It is known that there is a connection-preserving diffeomorphism $\bar{M} \cong G,[5]$, [7], [9]. Let $\pi$ be the group of covering transformations, so that $M \cong \pi \backslash G$. $\pi$ is a properly discontinuous and fixed-point free group of connection-preserving diffeomorphisms of $G$ and hence $\pi \subset A(G)$ by Proposition 2.1. Thus the proof is finished.

Remark 3.4. The question arises which Lie groups $G$ do admit discrete subgroups $\pi \subset A(G)$ acting properly discontinuously on $G$ such that $\pi \backslash G$ is compact. This question will not be treated here. However, the answer is certainly affirmative for those Lie groups which admit discrete subgroups with compact quotient. This is known to be the case for semi-simple Lie groups (A. Borel, Compact Clifford-Klein forms of symmetric spaces, Topology 2 (1963) 111-122) and nilpotent Lie groups with rational structural constants (A. Malcev, On a class of homogeneous spaces, Izv. Akad. Nauk SSSR Ser. Mat. 13 (1949) 9-32; Amer. Math. Soc. Transl. No. 39 (1951)).

Remark 3.5. That the completeness in the sense used here does not imply that any two points can be joined by a geodesic can be seen as follows. On a group manifold the geodesics through $e$ are the 1-parameter subgroups of $G$. It suffices to exhibit a $G$ where the exponential map exp is not surjective, e.g. on $\operatorname{SL}(2, R)$ the matrix $\left(\begin{array}{rc}-2 & 0 \\ 0 & -1 / 2\end{array}\right)$ is not square, and hence certainly not in the image of exp.

The Structure Theorem 3.3 has the following analogue for complex flat manifolds, which can be proved in the same way.

Theorem 3.6. Let $M$ be a connected complex manifold. Then $M$ admits a complete flat complex analytic connection with parallel torsion if and only if there are a connected and simply connected complex Lie group $G$, a subgroup $\pi$ of the complex affine group of $G$ acting properly discontinuously and without fixed points on $G$, and a connection-preserving holomorphism $M \cong \pi \backslash G$.

Examples to which Theorem 3.6 applies are compact complex parallelizable manifolds or more generally compact hermitian manifolds whose hermitian connection is complex analytic; these manifolds are then necessarily flat, complete and have parallel torsion, as proved in [3] and [11]. Theorem 3.6 then applies and provides a slight improvement of Theorem 4 of [3] to the 
effect that $\pi=\pi_{1} M$ is actually a discrete subgroup of the affine holomorphic group of the complex Lie group $G=\bar{M}$.

4. Let $M$ be a connected manifold with complete flat connection and parallel torsion. We propose to study the homotopy groups of $M$.

Proposition 4.1. $\pi_{2} M=0$.

Proof. By Structure Theorem 3.3 the universal covering is the fibration $\pi \rightarrow G \rightarrow M$ with a Lie group $G$. The classical result $\pi_{2} G=0$ and the exact homotopy sequence imply the Proposition.

Concerning the next homotopy group we have.

Theorem 4.2. $\pi_{3} M=0$ if and only if $M$ is an Eilenberg-MacLane space of type $K(\pi, 1)$.

Proof. Assume $\pi_{3} M=0$. Then for the group $G$ in $\pi \rightarrow G \rightarrow M$ we have $\pi_{3} G=0$. It is to show that $G$ is contractible. Let $K \subset G$ be a maximal connected compact subgroup of $G$. By Iwasawa, $G / K$ is homeomorphic to $R^{d}$. It suffices to show that $K=\{e\}$.

The Lie algebra $\mathfrak{k}$ of $K$ is reductive: $\mathfrak{t}=[\mathfrak{k}, \mathfrak{k}] \oplus \mathfrak{z}, z$ being the center of $\mathfrak{t}$. $K$ is simply connected, and hence is the product $K^{\prime} \times Z$ of a semi-simple Lie group $K^{\prime}$ with Lie algebra $\mathfrak{f}^{\prime}=[\mathfrak{f}, \mathfrak{t}]$ and a commutative Lie group $Z$ with Lie algebra $z$. Since the compactness of $K$ implies that of $Z, Z$ is a torus. But $K$ is simply connected and so is $Z$. This shows $Z=\{e\}$ and $K=K^{\prime}$ is compact and semi-simple.

We show $K=\{e\}$ by contradiction. Assuming $K \neq\{e\}$, it follows by a classical result of $E$. Cartan that $H^{3}(K, R) \neq 0$ and hence also $H_{3}(K, Z) \neq 0$ by the universal coefficient theorem. Since $\pi_{1} K=\pi_{2} K=0$ we have by Hurewicz $\pi_{3} K \cong H_{3}(K, Z) \neq 0$. But $\pi_{3} G=0$ implies $\pi_{3} K=0$, as $G / K$ is homeomorphic to $\boldsymbol{R}^{d}$. This contradiction finishes the proof of Theorem 4.2.

Complete flat manifolds without torsion correspond to the case $G=R^{n}$ and are certainly of type $K(\pi, 1)$. A description of the class of groups $G$ occurring as universal covering groups in Theorem 4.2 is given in

Proposition 4.3. Let $\pi \rightarrow G \rightarrow M$ be the universal covering of a connected manifold $M$ with complete flat connection and parallel torsion. Then $M$ is of type $K(\pi, 1)$ if and only if $G$ is homeomorphic with $\boldsymbol{R}^{d}$.

Proof. This is clear from the proof of Theorem 4.2.

Remark 4.4. If $M$ is of type $K(\pi, 1)$ as in Proposition 4.3 , then $\pi$ has no element of finite order. In fact, the universal covering $G$ is an acyclic manifold. But it is known that no finite group can act properly discontinuously and without fixed points on such a manifold [6, p. 357].

Observe also that if $M$ is of type $K(\pi, 1)$ as in Proposition 4.3 , then the universal covering $\pi \rightarrow G \rightarrow M$ is a universal $\pi$-bundle.

A sufficient condition for $M$ to be of type $K(\pi, 1)$ is given in

Theorem 4.5. Let $\pi \rightarrow G \rightarrow M$ be the universal covering of a connected manifold $M$ with complete flat connection and parallel torsion. If $G$ is solvable, then $M$ is of type $K(\pi, 1)$. 
Proof. Let $\mathfrak{r}$ be the radical of $g$. Then

$$
0 \rightarrow \mathfrak{x} \rightarrow \mathrm{g} \rightarrow \mathrm{g} / \mathrm{x} \rightarrow 0
$$

splits and the image of a splitting is a Levi subalgebra $\mathfrak{g}$ of $\mathfrak{g}$, [4]. $g$ is solvable if $g=x$, i.e. if and only if $\mathfrak{g}=0$ for some (and hence any) Levi subalgebra $\mathfrak{s}$ of $\mathfrak{g}$.

Let $\mathfrak{f} \subset \mathrm{g}$ be the Lie algebra of a maximal compact subgroup $K \subset G$. From the proof of Theorem 4.2 it follows that $\mathfrak{t}$ is semi-simple. Let $\mathfrak{s} \subset \mathfrak{g}$ be a maximal semi-simple subalgebra containing $\mathfrak{t}: \mathfrak{f} \subset \mathfrak{g}$. Then $\mathfrak{g}$ is a Levi subalgebra of $\mathrm{g}$. If $\mathrm{g}$ is solvable, $\mathfrak{g}=0$ and therefore $\mathfrak{t}=0$, which shows that $G$ is homeomorphic with $R^{d}$ and $M$ is of type $K(\pi, 1)$ by Proposition 4.3.

We conclude with the observation that the solvability of $G$ can be expressed purely as a condition on the torsion $T$ of the connection on $M$. In fact, for the Lie algebra $g$ of the invariant vector fields on $\bar{M}$ we have $T(X, Y)$ $=-[X, Y]$ as mentioned above. Hence $\mathrm{g}$ is solvable if and only if the sequence

$$
T(X, Y), T\left(T(X, Y), T\left(X^{\prime}, Y^{\prime}\right)\right), \cdots
$$

leads to an expression which is identically zero; being local, this condition can also be expressed on $M$ by locally invariant vector fields $X, Y, \cdots$, and therefore, by the preceding theorem, is a sufficient condition for a manifold with a complete flat connection and parallel torsion to be of type $K(\pi, 1)$.

\section{References}

[1] L. Auslander, A fixed point theorem for nilpotent Lie groups, Proc. Amer. Math. Soc. 9 (1958) 822-823.

[2] L. Auslander \& L. Markus, Holonomy of flat affinely connected manifolds, Ann. of Math. 62 (1955) 139-151.

[3] M. W. Boothby, Hermitian manifolds with zero curvature, Mich. Math. J. 5 (1958) 229-233.

[4] N. Bourbaki, Algèbres de Lie, Hermann, Paris, 1960.

[5] E. Cartan, La géométrie des groupes de transformations, J. Math. Pures Appl. 6 (1927) $1-119$.

[6] H. Cartan \& S. Eilenberg, Homological algebra, Princeton University Press, Princeton, 1956.

[7] W. Greub, Liesche Gruppen und affin zusammenhängende Mannigfaltigkeiten, Acta Math. 106 (1961) 65-111.

[8] S. Helgason, Differential geometry and symmetric spaces, Academic Press, New York, 1962.

[9] N. Hicks, A theorem on affine connections, Illinois J. Math. 3 (1959) 242-254.

[10] F. Kamber \& P. Tondeur, Flat bundles and characteristic classes of group representations, Amer. J. Math. 89 (1967) 857-886.

[11] H. C. Wang, Complex parallisable manifolds, Proc. Amer. Math. Soc. 5 (1954) 771-776.

INSTITUTE FOR ADVANCED STUDY

WESLEYAN UNIVERSITY

UNIVERSITY OF ILLINOIS 
